\title{
Disección arterial cérvico-cefálica post trauma en pediatría
}

\section{Cervicocephalic arterial dissection post trauma in children}

\section{Resumen}

Valentina Kobus', Belén Giménez', Javier Carcey², José Ignacio Marín³

Introducción: la disección arterial cérvico-cefálica (DACC) es una causa importante de accidente cerebrovascular (ACV) en pediatría. Dentro de los factores de riesgo más relevantes están los traumas cervicales y cefálicos, presentes en el $50 \%$ de los casos. El pronóstico es variable y depende de la magnitud de oclusión de la arteria afectada. Es importante la detección precoz de esta patología, que muchas veces se presenta con pocos síntomas en pediatría, lo que genera retraso en el diagnóstico y tratamiento. Objetivo: presentar la evidencia disponible sobre DACC incluyendo DACC post trauma para familiarizar a los médicos generales y especialistas sobre la sospecha diagnóstica, diagnóstico enfocado en neuroimágenes y posibles manejos de esta patología. Métodos: se llevó a cabo una revisión bibliográfica de la literatura científica sobre esta condición. Dentro de los criterios de selección de los estudios se consideró la fecha de publicación, el diseño y la relevancia. Conclusiones: la DACC post trauma es una causa frecuente de ACV en pediatría. Se puede presentar con escasa sintomatología, por lo que es importante mantener una alta sospecha en contexto de trauma cervical o cefálico, implementando un diagnóstico y tratamiento precoces para mejorar el pronóstico de los pacientes.

Palabras clave: disección arterial; disección carotídea; disección arterial cérvico-cefálica; trauma cervical; accidente cerebrovascular; pediatría.

\begin{abstract}
Introduction: Craniocervical arterial dissection (CCAD) is a major cause of arterial ischemic stroke (AIS) in children. The most important risk factors are cervical and cephalic traumas, present in 50\% of all cases. The prognosis changes depending on the magnitude of the artery affected. Early detection of this pathology is important. It many times presents itself with few symptoms, which generates a delay in its diagnosis and treatment. Objective: To present the evidence available on CCAD, including CCAD post-trauma, to familiarize general physicians and specialists with this diagnosis, neuroimages required, and possible treatments. Methods: Extensive revision of bibliographic scientific literature about this condition. The criteria selection to include studies in this review were the date of publication, the design of the study, and their relevance. Conclusions: the CCAD post-trauma is a significant cause of AIS in children. It can present itself with mild symptomatology, which is why it is relevant to suspect it in the context of cervical or cephalic trauma, aiming for an early diagnosis and treatment to improve the outcome of patients.
\end{abstract}

Keywords: arterial dissection; carotid dissection; cervicocephalic arterial dissection; cervical trauma; stroke; pediatrics.

Fecha de envío: 2020-05-19 - Fecha de aceptación: 2021-06-09

\section{Introducción}

Las disecciones arteriales cérvico-cefálicas (DACC) son una causa relevante de accidentes cerebrovasculares (ACV) isquémicos en pediatría, responsables del 7,5 - 20\% de estos (Lin et al., 2010; Simsek et al., 2020). Según el International Pediatric Stroke Study (IPSS), un 53\% de los pacientes con diagnóstico de ACV tuvieron como factor de riesgo una patología arterial, dentro de las cuales un
$20,2 \%$ fueron disecciones arteriales. Esto corresponde a un $10,7 \%$ del total de los factores de riesgo asociados a ACV isquémicos en pediatría (Mackay et al., 2011). Lo anterior toma suma relevancia ya que los ACV isquémicos se han vuelto una causa significativa de morbilidad neurológica en pediatría, principalmente debido a la baja sospecha diagnóstica que se tiene de esta patología, lo que lleva a grandes repercusiones a nivel personal, familiar, económico y social (Mackay et al., 2011).

(1) Facultad de Medicina, Pontificia Universidad Católica de Chile.

(2) Departamento de Pediatría, Complejo Asistencial Dr. Sótero del Río, Santiago, Chile.

(3) Departamento de Neurología Pediátrica, Complejo Asistencial Dr. Sótero del Río, Santiago, Chile.

Autor de correspondencia: javier.carcey@ssmso.cl 
Kobus et al.

Las DACC corresponden a la formación de un hematoma en la pared de una arteria cervical o intracraneal (Debette et al., 2015) debido a la pérdida de integridad del vaso sanguíneo, habitualmente por separación de la íntima de la pared arterial. Se pueden diferenciar según el lugar de la disección: las subintimales que disminuyen el calibre del vaso al desplazar el endotelio hacia el lumen, y las sub adventiciales que forman aneurismas disecantes que en niños frecuentemente aparecen en la circulación vertebral C1-C2 y comprimen los sitios rodeando la disección. Mientras que en los adultos el riesgo de DACC es mayor por la presencia de aterosclerosis, en niños se da por una musculatura cervical inmadura que contribuye a una baja estabilidad craneocervical y lleva a mayor dependencia de estructuras ligamentosas. Además, se suma la gran proporción cefálico-corporal que se da en los niños y no así en los adultos (Hejrati et al., 2020).

La incidencia anual estimada de las DACC corresponde a 2,5 en 100000 niños, no obstante, varía según etnia y grupo etario (Stence et al., 2011). La incidencia es mayor en hombres, con predominio intracraneal, y en comparación a los adultos hay mayor incidencia de disecciones espontáneas (Lin et al., 2010). Dentro de los factores de riesgo más relevantes están los traumas cervicales, presentes en aproximadamente el 50\% de los casos y favorecidos por traumas de alta energía como por ejemplo accidentes automovilísticos, en bicicleta, o agresiones físicas. La displasia fibromuscular está presente en 15-20\% de las disecciones. Existen otros factores los cuales se describen en la Tabla 1 (Simsek et al., 2020) (Tabla 1). El pronóstico puede ser variable y depende de la magnitud de oclusión de la arteria afectada (Debette et al., 2015).

Tabla 1: Factores predisponentes de DACC.

Traumas cervicales.

Displasia fibromuscular.

Enfermedades del tejido conectivo: Síndrome de Ehler-Danlos, Enfermedad de Marfan.

Enfermedad de Moyamoya.

Deficiencia de alfa-1 antitripsina.

Infecciones faríngeas.

Ateroesclerosis.

Homocisteinuria.

Anticuerpos antifosfolípidos.

Malformaciones vasculares.

\section{Aproximación diagnóstica}

\section{Cuadro clínico}

El cuadro clínico de las DACC en pediatría puede ser muy variable, por lo que es muy importante mantener una alta sospecha clínica en pacientes jóvenes con síntomas neurológicos y antecedente de trauma cervical y/o cefálico (Jariwala et al., 2011). Es importante mencionar que, si bien el reconocimiento del ACV en pediatría ha mostrado mejoras, la mediana de tiempo reportada desde el inicio del síntoma hasta la confirmación diagnóstica es de 25 horas (Shin et al., 2016). Esto tiene importancia en la práctica clínica, ya que se ha visto que un diagnóstico temprano con inicio de terapia apropiada antes del inicio de síntomas neurológicos es importante en términos de pronóstico a largo plazo (Orman et al., 2014).

La detección de las DACC post trauma antes del inicio de los síntomas es esencial, dado que en población pediátrica los signos clínicos de isquemia están raramente presentes o pueden ser malinterpretados. Sin síntomas de isquemia, la presencia de una disección arterial puede ser difícil de detectar. Se ha descrito la ocurrencia de un periodo latente asintomático que puede variar ampliamente, desde minutos a meses, ocasionalmente años, antes de que el cuadro presente síntomas. Los síntomas iniciales pueden ser sutiles y son dependientes del territorio vascular afectado y por lo mismo son heterogéneos (Jariwala et al., 2011). Los síntomas y signos más comunes descritos en la literatura son hemiparesias, cefalea, afasia, disfasia, anopsia y alteración del nivel de conciencia (Cebeci et al., 2018), de estos la cefalea cervical persistente un síntoma bastante orientador de disección. Algunos pacientes pueden presentar síntomas que orientan hacia diagnósticos imitadores de ACV como migraña, encefalitis y tumores, que son diagnósticos más probables que el ACV (Shin et al., 2016). Si bien la ocurrencia de síndrome de Horner en población pediátrica suele ser poco frecuente, se debe mantener una alta sospecha en cuadros con estos síntomas y antecedente de trauma, ya que esta podría ser la única manifestación (Hejrati et al., 2020).

\section{Diagnósticos diferenciales}

Es importante tener en cuenta, como se mencionó previamente, que los cuadros de ACV en población pediátrica pueden presentar síntomas variados y poco específicos, por lo que es muy importante mantener una alta sospecha clínica.

En primer lugar, se debe tener en cuenta el diagnóstico diferencial del ACV con los llamados "imitadores de ACV" (Tabla 2), los cuales se pueden presentar con cuadros similares y retrasar el diagnóstico y tratamiento. 
Kobus et al.

Tabla 2: Diagnósticos diferenciales clínicos de ACV (Stroke mimics).

Ataque isquémico transitorio (TIA).

Síndrome de encefalopatía posterior reversible (PRES).

ACV hemorrágico: rotura aneurismática, malformación arteriovenosa (MAV).

Traumatismo encéfalo-craneano (TEC),

Vasoconstricción cerebral reversible.

Enfermedades metabólicas: encefalomiopatía mitocondrial, acidosis láctica y episodios parecidos a ACV (MELAS), homocisteinuria, déficit de n-glicosilación, acidurias orgánicas, enfermedad de Fabry.

En la literatura se describen diferentes diagnósticos diferenciales para cuadros de DACC, los que se pueden agrupar como se muestra en la Tabla 3. Además, debido a la incidencia de ACV asociado a DACC, se vuelve relevante identificar otras causas de ACV como se muestra en la Tabla 4.

Tabla 3: Diagnóstico diferencial de DACC con otras arteriopatías.

No inflamatorias: Anemia de células falciformes, displasia fibromuscular, moyamoya, vasoespasmo secundario a hemorragia subaracnoidea, estenosis ateroesclerótica.

Inflamatorias: Arteriopatía cerebral focal, vasculitis, arteriopatía post varicela.

Variaciones anatómicas.

Arteriopatías no especificadas.

Tabla 4: Diagnósticos diferenciales de DACC con otras causas de ACV.

Arteriopatías.

Patologías agudas de cabeza y cuello: Meningitis, faringitis, TEC, otitis media aguda, mastoiditis, sinusitis, cirugía intracraneal reciente.

Patologías crónicas de cabeza y cuello: Migraña, tumor cerebral, shunt ventriculoperitoneal, aneurisma cerebral, MELAS, síndrome de PHACES, malformación arteriovenosa intracraneal.

Estados protrombóticos.

Condiciones cardiacas: enfermedad cardiaca congénita y adquirida, cateterización cardiaca, arritmias.

Condiciones sistémicas agudas: sepsis, shock, deshidratación, fiebre $>48$ horas.

Condiciones sistémicas crónicas: Deficiencia de hierro, uso de anticoncepción oral, trisomía 21, neoplasia hematológica, enfermedades del tejido conectivo, anemia falciforme, uso de catéter permanente.
Recomendamos un enfrentamiento ordenado, basado en la historia clínica, examen físico y neuroimágenes, recalcando la importancia de mantener una alta sospecha clínica.

\section{Neuroimágenes}

Las neuroimágenes poseen un rol esencial en establecer el diagnóstico de DACC post trauma y sus complicaciones. Los neuro radiólogos deben ser conscientes del riesgo aumentado de DACC posterior a un trauma de cabeza y cuello en población pediátrica debido a las propiedades biomecánicas de la unión craneocervical y la movilidad del cuello (Orman et al., 2014).

\section{Tomografía computarizada (TC) cerebral sin contraste}

Si bien en la literatura la TC cerebral sin contraste se describe como poco sensible para detectar DACC, entrega información sobre fracturas de la base del cráneo, lo que a su vez debería generar sospecha de DACC ya que se ha visto que se asocian comúnmente ambas condiciones (Orman et al., 2014). Cabe mencionar que en el momento de la lesión inicial la mayoría de las imágenes resultan normales, lo que podría favorecer el retraso en el diagnóstico (Steinborn et al., 2011). En resumen, es una herramienta de gran utilidad para el diagnóstico diferencial con lesiones traumáticas. A pesar de lo mencionado anteriormente, se utiliza frecuentemente como imagen inicial dada la amplia disponibilidad y el bajo costo. Una de sus limitaciones importantes es el efecto nocivo de la radiación ionizante en el desarrollo de estructuras cerebrales y radiosensibles. Se ha visto que el riesgo de sufrir efectos secundarios por radiación es mayor en población pediátrica que en los adultos, además de que tienen más años de vida esperables durante los cuales se podría desarrollar un cáncer inducido por radiación (Brenner \& Hall, 2007). Por lo expuesto anteriormente se deben poner en perspectiva los beneficios y los riesgos de este examen.

\section{AngioTC}

Estudios recientes reportan gran sensibilidad para esta imagen (98-100\%) en el diagnóstico de DACC espontánea y traumática en adultos. Otras ventajas son su amplia disponibilidad, relativo bajo costo, carácter no invasivo y la posibilidad de solicitarlo como estudio inicial en contexto de trauma. La gran desventaja del AngioTC, especialmente en pediatría, es el uso de radiación ionizante. Además, esta modalidad aún no ha sido ampliamente estudiada para el diagnóstico de DACC en población pediátrica (Stence et al., 2011).

\section{Angiografía convencional}

Esta modalidad es útil para definir los cambios vasculares en la DACC y se ha considerado por décadas como el estándar de 
Kobus et al.

referencia para el diagnóstico con sensibilidad de 98-100\% (Fleck et al., 2011; Stence et al., 2011). Los hallazgos angiográficos específicos en DACC incluyen la identificación de colgajos intimales, doble lumen vascular, formación de bolsa aneurismática, estenosis, y oclusión a menudo descrita en forma de llama (Lin et al., 2010). En comparación con los adultos, esta modalidad posee ciertas desventajas en población pediátrica: dificultad y morbilidad asociada al acceso vascular arterial, necesidad de anestesia general, el uso de radiación ionizante y el alto costo en comparación con otras modalidades de neuroimágenes (Hejrati et al., 2020). Es por esto que en los últimos años algunos centros la han ido reemplazando por técnicas más nuevas y en general no invasivas (Chabrier et al., 2003; Rafay et al., 2006; Mortazavi et al., 2011).

\section{Resonancia magnética cerebral}

Se ha descrito que tiene una sensibilidad y especificidad similares a la angiografía convencional (Chamoun et al., 2008). Sus beneficios en comparación con la angiografía convencional son mayor disponibilidad, menor costo y ser un estudio no invasivo. En general se postula su utilidad en combinación con Angio Resonancia Nuclear Magnética (RNM)(Jones et al., 2012), secuencia supresión grasa.

\section{Angioresonancia magnética cerebral}

Se ha descrito que tiene una sensibilidad y especificidad similares a la angiografía convencional (Chamoun et al., 2008). Dado los efectos deletéreos de la radiación ionizante en población pediátrica, esta modalidad ha tomado cada vez más rol diagnóstico en múltiples centros, a pesar de que algunos pacientes necesitan sedación. Algunos autores postulan que este sería el nuevo estándar de referencia, pero esto se mantiene como punto de controversia (Hejrati et al., 2020). En múltiples estudios y revisiones se postula que actualmente la combinación de RMN con Angio RNM son una herramienta fundamental en el diagnóstico de esta patología (Jones et al., 2012).

\section{Ecografía Doppler}

Algunos trabajos han postulado el uso de ecografía Doppler en el diagnóstico de DACC, pero esto no ha sido ampliamente estudiado ni validado en población pediátrica. Además, posee una sensibilidad muy variable, dependiente del operador (Stence et al., 2011).

Si bien el uso de neuroimágenes es muy importante para el diagnóstico de DACC, para la selección de una modalidad se deben tener en cuenta sus beneficios y potenciales riesgos, además de los costos y accesibilidad de cada centro. Actualmente no se cuenta con un protocolo único de enfrentamiento, por lo que postulamos que cada centro debiese tener sus directrices.

\section{Tratamiento}

\section{Opciones de tratamiento}

El tratamiento de la DACC puede variar dependiendo de si la disección es intra o extracraneana. A grandes rasgos, los dos pilares son el tratamiento médico y el tratamiento quirúrgico o endovascular. El manejo de las DACC extracraneales puede variar incluso entre subespecialidades y debido a ausencia de consenso en el tratamiento el manejo se aleja de ser óptimo en pediatría (Wang et al., 2016). Lo mismo ocurre con las con disecciones intracraneales ya que no existen ensayos randomizados y solo hay estudios observacionales con muestras pequeñas de pacientes (Debette et al., 2015).

\section{Tratamiento médico}

El tratamiento médico en una DACC extracraneal consiste en manejo de soporte que incluye principalmente la prevención de eventos tromboembólicos a través de la antiagregación o anticoagulación. Las guías clínicas del año 2008 de ACV infantil publicadas por el Consejo de ACV de la American Heart Association y el Consejo de Enfermedad Cardiovascular en el joven, recomiendan tratamiento con anticoagulación, ya sea heparina no fraccionada, heparina de bajo peso molecular, o warfarina con duración de mínimo 3 meses, hasta 6 meses (Roach et al., 2008). Según la octava guía clínica de la Universidad Americana de médicos de tórax, los casos de ACV isquémicos debido a DACC en neonatos o niños la anticoagulación debe mantenerse por al menos 6 semanas (Monagle et al., 2008). Un metaanálisis de Cochrane del año 2010 que incluyó 36 estudios observacionales no encontró diferencia significativa entre el uso de antiagregación plaquetaria y anticoagulación en cuanto a mortalidad, incidencia de ACV, y discapacidad por lo que las guías clínicas plantean su equivalencia (Lyrer et al., 2010). Las dosis de aspirina y clopidogrel no han sido establecidas en base a población pediátrica, sino que se extrapolan de la literatura de adultos (Wang et al., 2016). Las guías clínicas para adultos de la American Heart Association y American Stroke Association del año 2018 recomiendan que para disecciones carotídeas extracraneales y vertebrales es razonable el tratamiento con anticoagulación o antiagregación con una duración de tres a seis meses (Powers et al., 2018). El tratamiento con anticoagulación no está recomendado en niños con disección intracraneal, con evidencia clase $C$, según la guía pediátrica de la AHA 2008. Sin embargo, esta recomendación debe ser sometida a mayor discusión en base a las nuevas recomendaciones para adultos en la guía AHA 2018 (Nash et al., 2019).

Es importante destacar que el uso de antiagregación o anticoagulación en DACC involucra un riesgo de sangrado inherente por lo que se recomienda iniciar tratamiento luego de la transformación 
Kobus et al.

hemorrágica o en la fase de resangrado de la lesión cerebral traumática (Hejrati et al., 2020). En un escenario de disección por politrauma se contraindica tratamiento con anticoagulación y el paciente se beneficiaría de imágenes seriadas o vigilancia neurológica intensiva para evaluación constante de deterioro del estado neurológico (Wang et al., 2016). En una serie un $42 \%$ de los pacientes pediátricos con lesión cerebrovascular aguda se trataron con algún tipo de tratamiento médico, no se observaron complicaciones hemorrágicas (Dewan et al., 2016). Además, un reporte de casos de niños con disección arterial cérvico-cefálicas post traumáticas con síntomas neurológicos mostró mayor riesgo de persistencia o agravación de déficit neurológicos cuando se retrasa el inicio de la anticoagulación/agregación (Hejrati et al., 2020). Cabe destacar que el riesgo de eventos embólicos es mayor en cuanto se produce la lesión vascular y luego la primera semana de la injuria. Según la literatura, hasta un $85 \%$ de las disecciones carotídeas de causa espontánea mejoran con el uso de terapias antitrombóticas (Wang et al., 2016). Importante considerar en cuanto al uso de aspirina en niños la posible aparición de síndrome de Reye, por lo que se podría considerar el uso de clopidogrel en estos casos (Wang et al., 2016). Por ende, la evidencia recopilada orientaría a la importancia de un inicio precoz de la anticoagulación o antiagregación siempre evaluando los riesgos versus beneficios del tratamiento en cada paciente y tomando en cuenta que poco se sabe del uso a largo plazo del uso de estos fármacos en niños.

Según la guía clínica de ACV neonatal e infantil publicadas por el Consejo de ACV de la American Heart Association (AHA) en el año 2019, estos pacientes deben ser manejados en Unidades de Cuidados Intensivos (UCI) al menos 24 horas. De esta manera se puede mantener control estricto de variables fisiológicas como lo son la presión arterial, la temperatura y la glicemia, para evitar aumentar el daño neurológico preexistente y disminuir el riesgo de convulsiones y edema cerebral. Gran parte de la información que se usa actualmente para el manejo de pacientes pediátricos con ACV se extrapola de lo que tiene evidencia en adultos (Ferriero et al., 2019).

Con respecto al uso de anticoagulación en neonatos, la guía AHA 2019 establece que raramente se indica el uso de antiagregantes plaquetarios como la aspirina o de anticoagulantes como la heparina de bajo peso molecular (HBPM) y la heparina no fraccionada (HNF) por el bajo riesgo de recurrencia de ACV. Sin embargo, debe considerarse su uso en neonatos con alto riesgo de recurrencia de ACV como lo son tener una trombofilia documentada o una patología cardiaca congénita. Se plantea que, en ausencia de ensayos clínicos, hasta el momento la anticoagulación parece ser segura para neonatos con infartos venosos en contexto de trombosis de seno venoso, especialmente en ausencia de hemorragia cerebral
En niños mayores con ACV generalmente el manejo inicial es con antiagregantes (aspirina) o anticoagulantes (HBPM o HNF) a menos que exista una contraindicación evidente para su uso. Aún es controversial si existe mayor beneficio de la anticoagulación o de la antiagregación, no obstante, ambos poseen perfiles de seguridad similares. En casos de ACV por causas cardiacas o trombofilias se prefiere el uso de anticoagulantes. Además, se plantea que el uso a largo plazo de tratamiento antitrombótico, antiagregantes o anticoagulantes (HBPM o warfarina) podría prevenir la recurrencia tardía de ACV, dependiendo de la etiología. Por lo general la profilaxis secundaria se mantiene por al menos 2 años, pero en la mayoría de los casos incluso por más tiempo, sin embargo, aún se desconoce el tiempo exacto por el cual se debería mantener el tratamiento antitrombótico (Ferriero et al., 2019).

\section{Tratamiento endovascular y cirugía}

Los pacientes con disección intracraneal con hemorragia subaracnoidea por lo general son de resolución quirúrgica o a través de procedimiento endovascular. Hay un riesgo de resangrado de hasta un 40\% dentro del primer día posterior al evento (Lin et al., 2010). Debido a los grandes avances de la tecnología endovascular, actualmente el enfoque terapéutico se centra más en esta alternativa que en las técnicas de microcirugía. Además, los bypass quirúrgicos aparte de ser más complejos y consumir mucho tiempo, tienen una elevada tasa de mortalidad (Wang et al., 2016).

El objetivo del tratamiento endovascular es restablecer el flujo del vaso fuente y ocluir el pseudoaneurisma para disminuir el riesgo futuro de secuelas neurológicas. No obstante, la literatura en cuanto a stents intracraneales en pediatría es escasa. Un número limitado de reporte de casos ha demostrado éxito al utilizar stents en aneurismas fusiformes y en casos de pseudoaneurismas originados por trauma donde se requiere reconstrucción del vaso lesionado (Wang et al., 2016). Dada esta evidencia el uso de tratamiento endovascular se reserva para casos de aneurismas sintomáticos o en crecimiento, fístulas arteriovenosas asintomáticas, estenosis arteriales sintomáticas, disecciones intracraneales asociadas a hemorragia, o respuesta refractaria a tratamiento médico (Hejrati et al., 2020). Uno de los desafíos del uso de stent neurovasculares en niños es la elección del tamaño del stent el cual debe ser elegido cuidadosamente ya que los vasos intracraneales muchas veces no han alcanzado su máximo diámetro y pueden crecer aproximadamente hasta los 4-5 años en el caso de la arteria carótida interna y arteria cerebral anterior. La arteria cerebral media según los mismos estudios, alcanzaría su máximo diámetro aproximadamente a los 6 meses de vida. Poner un stent muy grande para el tamaño del vaso puede contribuir a que en el futuro se estenose el lumen. Además, se suma que, como ocurre también en los adultos, el 
Kobus et al.

poner un stent aumenta el riesgo de estrechamiento endotelial a lo largo de los años, lo que contribuye a estenosis en pacientes con larga expectativa de vida (Wang et al., 2016).

La Guía AHA Pediátrica del 2019 sugiere con respecto al manejo de ACV que en ausencia de ensayos clínicos el uso de terapias hiperagudas sigue siendo controversial. Las terapias hiperagudas incluyen el uso intravenoso de activador tisular del plasminógeno (tPA), el uso intraarterial de tPA o la trombectomía endovascular. Se plantea como razonable incluir en estas terapias a niños que cumplan con criterios como: déficit neurológico persistente, confirmación radiográfica de gran oclusión arterial, ser niños de gran tamaño, que la decisión de tratamiento sea en conjunto con neurólogo experto, y la intervención realizada por un cirujano endovascular con experiencia en manejo de niños y además en realizar trombectomias en adultos. Se desconoce con certeza sobre la eficacia y seguridad de la aplicación de las terapias hiperagudas en ACV en niños. No existe evidencia que establezca una edad en la cual la trombectomía es segura para un niño, ni tampoco el tamaño del niño que sea más seguro (Ferriero et al., 2019).

\section{Manejos alternativos}

Se puede optar por manejo expectante en caso de que el riesgo de la intervención quirúrgica o endovascular sea muy alta o que el paciente esté basalmente muy frágil desde el punto de vista clínico (Debette et al., 2015). La evidencia sobre trombolisis en pediatría en ACV es limitada (Lin et al., 2010). El 2010 se inició el estudio Thrombolysis in Pediatric Stroke (TIPS) por parte del National Institute of Neurological Disorders and Stroke el cual acabó el 2013 por falta de pacientes agudos, sin resultados (Sánchez et al., 2017). Debido a las diferencias fisiopatológicas entre el ACV en niños y adultos resulta complejo utilizar la evidencia disponible para adultos y aplicarla en pediatría.

Durante los últimos años se plantea en la guía AHA pediátrica 2019 que ha surgido la tendencia a realizar hemicraniectomía descompresiva temprana en niños con infartos de gran volumen dentro de las primeras 24 horas de forma profiláctica o implementando una serie de imágenes y evaluación clínica las primeras 72 horas para monitorizar el edema y según eso evaluar la necesidad de una intervención quirúrgica. Sin embargo, falta evidencia para sugerir esto en todos los casos (Ferriero et al., 2019).

\section{Pronóstico}

El pronóstico de las DACC post trauma en pediatría es variable. Dependiendo de la extensión de la oclusión, el pronóstico varía entre recuperación completa de funciones hasta secuelas permanentes como hemiparesias severas, hemiplejías, afasia y convulsiones (Patel et al., 1995; Tabarki et al., 1997). Se vuelve necesario un control con imágenes a los 3-6 meses posterior al incidente para evaluar pronóstico. Las oclusiones totales de vaso tienen peor pronóstico en cuanto a recanalización versus las lesiones solo con flap intimal. Es importante identificar los casos en que una DACC completamente ocluida se convierte en una recanalización parcial posterior al inicio de tratamiento ya que el riesgo de tromboembolismo puede llegar a ser de un 50\% en estos pacientes (Hejrati et al., 2020). Para obtener buenos resultados a largo plazo es importante sospechar, diagnosticar e iniciar el tratamiento adecuado de manera rápida, idealmente antes del desarrollo de síntomas neurológicos graves, evitando el desarrollo de ACV secundario a la DACC (Cebeci et al., 2018). La mortalidad en pacientes con disecciones intracraneales con hemorragia subaracnoidea va desde un 19-50\%. Por otra parte, los pacientes sin hallazgos de hemorragia subaracnoidea tienen baja mortalidad, de aproximadamente un 3\%, parecido a los pacientes con disecciones de la arteria cervical (Debette et al., 2015).

\section{Conclusiones}

Las DACC secundarias a traumatismos de cabeza y cuello son una causa importante de ACV en pediatría, llegando a ser hasta el 20\% en algunas series. Esto es relevante ya que es una patología que puede causar daño neurológico permanente, por lo que es muy importante su pronto diagnóstico y tratamiento. Usualmente se diagnostica posterior a la aparición de los síntomas clásicos lo que empeora el pronóstico de los pacientes. Es por esto que planteamos la importancia de conocer esta patología, tanto para médicos generales como para especialistas. Si bien la aproximación diagnóstica suele ser mediante neuroimágenes, es importante recalcar que no hay un consenso sobre qué imagen utilizar en población pediátrica. Se postula que el estándar de referencia es la angiografía convencional, pero durante los últimos años han surgido alternativas menos invasivas, como la RNM o la angio RNM. Es por esto la importancia de estar familiarizados con las alternativas de los distintos centros y evaluar el costo y beneficio de cada uno de ellos. El manejo inicial puede variar dependiendo de si la disección es intra o extracraneana. En las DACC extracraneales consiste en el uso de antiagregación plaquetaria o anticoagulación ya que las guías clínicas plantean su equivalencia en cuanto a mortalidad, incidencia de ACV, y discapacidad. En las DAC intracraneales no se recomienda la anticoagulación ya que frecuentemente se asocian a hemorragias subaracnoideas. Dado lo anterior, los pacientes con disección intracraneal con hemorragia subaracnoidea por lo general son de resolución endovascular. El uso de stent para revascularización de disección carotídea y pseudoaneurismas ha demostrado ser una técnica factible y segura por lo que es el tratamiento de elección en lesiones refractarias a tratamiento médico o con contraindicación. Si bien existe gran cantidad de información científica disponible sobre esta patología, creemos que es importante seguir 
Kobus et al.

estudiando las diferentes técnicas de diagnóstico y tratamiento, para poder llegar a tener un consenso que disminuya el riesgo de secuelas a largo plazo en estos pacientes.

\section{Referencias:}

Brenner D. \& Hall EJ. (2007). Computed Tomography-an increasing source of radiation exposure. N Engl J Med. 357, 2277-2284.

Can F, Turhan S, Anil A, Karadem K. \& Isik O. (2019). A rare case of life-threatening traumatic carotid artery dissection in a child. General Thoracic and Cardiovascular Surgery 67, 483-485.

Cebeci D, Arhan, Demir E, Uçar M, Koç H, Serdaroğlu A. \& Öztürk Z. (2018). Internal carotid artery dissection without intracranial infarct following a minor shoulder trauma: The second pediatric case and review of the literature. J Clin Neuroscience. 56, 172-175.

Chabrier S, Lasjaunias P, Husson B, Landrieu P. \& Tardieu M. (2003). Ischaemic stroke from dissection of the craniocervical arteries in childhood: report of 12 patients. Eur J Paediatr Neurol. 7, 39-42.

Chamoun RB, Mawad ME, Whitehead WE, Luerssen TG. \& Jea A. (2008). Extracranial traumatic carotid artery dissection in children: a review of current diagnosis and treatment options. J Neurosurg Pediatr 2, 101-108.

Debette S, Compter A, Labeyrie M, Uyttenboogaart M, Metso T, Majersik J, Goeggel-Simonetti B, Engelter, S. \& Pezzini, A. (2015). Epidemiology, pathophysiology, diagnosis, and management of intracranial artery dissection. The Lancet Neurology 14, 640-654.

Dewan MC, Ravindra VM, Gannon S, Prather CT, Yang GL, Jordan LC, Limbrick D, Jea A, Riva-Cambrin J. \& Naftel RP. (2016). Treatment practices and outcomes after blunt cerebrovascular injury in children. Neurosurgery 79, 872-878.

Ferriero D, Fullerton $\mathrm{H}$, Bernard T, Billinghurst $\mathrm{L}$, Daniels S, DeBaun M, deVeber G, Ichord R, Jordan L, Massicotte P, Meldau J, Roach S. \& Smith E. (2019). Management of Stroke in Neonates and Children A Scientific Statement From the American Heart Association/ American Stroke Association. Stroke 50, e51-e96.

Fleck SK, Langner S, Baldauf J, Kirsch M, Kohlmann T. \& Schroeder HW. (2011). Incidence of blunt craniocervical artery injuries: use of whole-body computed tomography trauma imaging with adapted computed tomography angiography. Neurosurgery 69, 615-623.

Hejrati N, Ebel F, Guzman R. \& Soleman J. (2020). Posttraumatic cerebrovascular injuries in children. A systematic review. Child's Nervous System 36, 251-262.
Jariwala S, Crowley G. \& Roychowdhury S. (2011). Trauma-induced extracranial internal carotid artery dissection leading to multiple infarcts in a young girl. Pediatric Emergency Care 22, 737-742.

Jones T, Burlew C, Kornblith L, Biff W, Patrick D, Johnson J, Barnett C, Bensard D. \& Moore E. (2012). Blunt cerebrovascular injuries in the child. Am J Surg 204, 7-10.

Kulyk C, Farina F, Palmieri A, Viaro F, Causin F, Laverda A \& Baracchini C. (2017). "Phacing” a New Cause of Carotid Artery Dissection. The Neurologist 22, 54-56.

Lin J, Chou M, Lin K, Wong M. \& Wang H. (2007) Cerebral Infarct Secondary to Traumatic Carotid Artery Dissection. Pediatric Emergency Care 27, 166-168.

Lin Y, Feng-Chi C, Chung-Jung L, Ting-Chang H. \& Kao-Lun W. (2010). Endovascular Therapy in Pediatric Intracranial Carotid Artery Dissection. Pediatr Neurol. 42, 291-294.

Lyrer P. \& Engelter S. (2010). Antithrombotic drugs for carotid artery dissection. The Cochrane Database of Systematic Reviews 6, CD000255.

Mackay M, Wiznitzer M, Benedict S, Lee K, deVeber G. \& Ganesan V. (2011). Arterial Ischemic Stroke Risk Factors: The International Pediatric Stroke Study. ANN NEUROL 69, 130-140.

Monagle P, Chalmers E. \& Chan A. (2008). Antithrombotic therapy in neonates and children: American College of Chest Physicians Evidence-Based Clinical Practice Guidelines (8th edition). Chest $133,887 S-968 S$.

Moon K, Albuquerque F, Cole T, Gross B. \& McDougall C. (2017). Stroke prevention by endovascular treatment of carotid and vertebral artery dissections. J Neurolntervent Surg 9, 952-957.

Mortazavi MM, Verma K, Tubbs RS. \& Harrigan M. (2011). Pediatric traumatic carotid, vertebral and cerebral artery dissections: a review. Child's Nervous System 27, 2045-2056.

Nash M. \& Rafay MF. (2019). Craniocervical Arterial Dissection in Children: Pathophysiology and Management. Pediatric Neurology 95, 9-18.

Orman G, Tekes A, Poretti A, Robertson C. \& Huisman T. (2014). Post Traumatic Carotid Artery Dissection in Children: Not to be missed! J Neuroimaging 24, 467-472.

Patel H, Smith R. \& Garg B. (1995). Spontaneous extracranial carotid artery dissection in children. Pediatr Neurol 13, 55-60. 
Kobus et al.

Payton T, Siddiqui K, Sole D. \& McKinley D. (2004). Traumatic Dissection of the Internal Carotid Artery. Pediatr Emer Care 20, 27-29.

Powers W, Rabinstein A. \& Ackerson T. (2018). Guidelines for the early management of patients with acute ischemic stroke: a guideline for healthcare professionals from the American Heart Association/ American Stroke Association. Stroke 49, 46-110.

Rafay MF, Armstrong D, Deveber G, Domi T, Chan A. \& MacGregor DL. (2006). Craniocervical arterial dissection in children: clinical and radiographic presentation. J Child Neurol 21, 8-16.

Roach E, Golomb M, Adams R, Biller J, Daniels S, Deveber G, Ferriero D, Jones B, Kirkham F, Scott R. \& Smith E. American Heart Association Stroke Council; Council on Cardiovascular Disease in the Young. (2008). Management of stroke in infants and children: a scientific statement from a Special Writing Group of the American Heart Association Stroke Council and the Council on Cardiovascular Disease in the Young. Stroke 39, 2644-2691.

Rose E. \& Sherwin T. (2019). Carotid Dissection and Cerebral Infarction From Posterior Oropharyngeal Trauma. Pediatr Emer Care 35, e17-e21.

Sánchez M. (2017). Epidemiología y causas de la patología vascular cerebral en niños. Rev Esp Pediatr. 73, 1-5.

Shin J, Singh R. \& Carney M. (2016). Carotid Artery Dissection and Ischemic Stroke After Severe Coughing. Pediatr Emer Care 32, 693-695.
Simsek E, Yilmaz S, Oran I, Aktan G, Tekgul H. \& Gokben S. (2020). A rare cause of ischemic stroke in childhood: spontaneous long segment intracranial dissection. Child's Nervous System 36, 2871-2875.

Steinborn M, Schäffeler C, Kabs C, Kraus V, Rüdisser K. \& Hahn H. (2010). CT and MR imaging of primary cerebrovascular complications in pediatric head trauma. Emerg Radiology 17, 309-315.

Stence N, Fenton L, Goldenberg N, Armstrong-Wells J. \& Bernard T. (2011). Craniocervical Arterial Dissection in Children: Diagnosis and Treatment. Current Treatment Options in Neurology 13, 636-648.

Tabarki B, Madani A, Alvarez H, Husson B, Lasjaunias P, Landrieu P, Tardieu M. \& Sébire G. (1997). Accident vasculaire cérébral ischémique par dissection artérielle vertébrale. Arch Pediatr 4, 763-766.

Texakalidis P, Karasavvidis T, Giannopoulos S, Tzoumas A, Charisis N, Jabbour P, Machinis T, Rangel-Castilla L. \& Reavey-Cantwell J. (2019). Endovascular reconstruction of extracranial traumatic internal carotid artery dissections: a systematic review. Springer Nature 43, 931-940.

Wang A, Santarelli J. \& Stiefel M. (2016). Traumatic cervical internal carotid artery pseudoaneurysm in a child refractory to initial endovascular treatment: case report and technical considerations. Child's Nervous System 32, 2459-2464. 